\title{
Existence and nonexistence of entire $k$-convex radial solutions to Hessian type system
}

\section{Jixian Cui ${ }^{* *}$}

"Correspondence: jixiancui@126.com 'College of Science, Qiqihar University, Qiqihar 161006, P.R. China

\section{Springer}

\begin{abstract}
In this paper, a Hessian type system is studied. After converting the existence of an entire solution to the existence of a fixed point of a continuous mapping, the existence of entire $k$-convex radial solutions is established by the monotone iterative method. Moreover, a nonexistence result is also obtained.
\end{abstract}

Keywords: $k$-convex radial solution; Existence; Nonexistence; Hessian type system

\section{Introduction}

In this paper, we study the existence of entire $k$-convex radial solutions to the following problem of Hessian type system:

$$
\begin{cases}\sigma_{k}\left(\lambda\left(D^{2} u+\mu|\nabla u| I\right)\right)=p(|x|) f_{1}(u) f_{2}(v), & x \in B_{1}(0), \\ \sigma_{l}\left(\lambda\left(D^{2} v+v|\nabla v| I\right)\right)=q(|x|) g_{1}(u) g_{2}(v), & x \in B_{1}(0), \\ u=v=0, & x \in \partial B_{1}(0),\end{cases}
$$

where $k, l=1,2, \ldots, N, \mu, v \geq 0$ are constants, $B_{1}(0)$ is the unit ball in $\mathbb{R}^{N}$, for any $N \times N$ real symmetric matrix $A, \lambda(A)$ denotes the eigenvalues of $A, D^{2} u(x)=\left(\frac{\partial^{2} u(x)}{\partial x_{i} \partial x_{j}}\right)$ denotes the Hessian matrix of the function $u \in C^{2}\left(\overline{B_{1}(0)}\right), \nabla u$ denotes the gradient of $u$, and $\sigma_{k}(\lambda)=\sum_{1 \leq i_{1}<\cdots<i_{k} \leq N} \lambda_{i_{1}} \cdots \lambda_{i_{k}}$ denotes the $k$ th elementary symmetric function of $\lambda=$ $\left(\lambda_{1}, \ldots, \lambda_{N}\right) \in \mathbb{R}^{N}$.

For $p, q, f_{1}, f_{2}, g_{1}, g_{2}$, we introduce the following conditions:

(H1) $p, q \in C([0,1],(0,+\infty)) . f_{1}, f_{2}, g_{1}, g_{2} \in C((-\infty, 0],[0,+\infty))$ are decreasing.

(H2) For any $a>0$, the integral $\int_{-\infty}^{-a} \frac{d \tau}{\left(f_{1}(\tau) f_{2}(\tau)\right)^{\frac{1}{k}}+\left(g_{1}(\tau) g_{2}(\tau)\right)^{\frac{1}{l}}}$ is divergent.

(H3) For any $a>0$, the integral $\int_{-a}^{0} \frac{d \tau}{\left(f_{1}(\tau) f_{2}(\tau)\right)^{\frac{1}{k}}+\left(g_{1}(\tau) g_{2}(\tau)\right)^{\frac{1}{l}}}$ is divergent.

Denote

$$
\Gamma_{k}:=\left\{\lambda \in \mathbb{R}^{N}: \sigma_{j}(\lambda)>0,1 \leq j \leq k\right\} .
$$

We say that a function $u \in C^{2}\left(\overline{B_{1}(0)}\right)$ is $k$-convex in $B_{1}(0)$ if $\lambda\left(D^{2} u(x)\right) \in \Gamma_{k}$ for all $x \in B_{1}(0)$.

(c) The Author(s) 2021. This article is licensed under a Creative Commons Attribution 4.0 International License, which permits use sharing, adaptation, distribution and reproduction in any medium or format, as long as you give appropriate credit to the original author(s) and the source, provide a link to the Creative Commons licence, and indicate if changes were made. The images or other third party material in this article are included in the article's Creative Commons licence, unless indicated otherwise in a credit line to the material. If material is not included in the article's Creative Commons licence and your intended use is not permitted by statutory regulation or exceeds the permitted use, you will need to obtain permission directly from the copyright holder. To view a copy of this licence, visit http://creativecommons.org/licenses/by/4.0/. 
In (1.1), if $\mu=0$ and $f_{2}(v) \equiv 1$, the first equation in the system becomes the following $k$-Hessian type equation:

$$
\sigma_{k}\left(\lambda\left(D^{2} u\right)\right)=p(|x|) f_{1}(u)
$$

if $\mu=v=0$ and $f_{1}(u)=g_{2}(v) \equiv 1$, the system becomes the following coupling $k$-Hessian system:

$$
\left\{\begin{array}{l}
\sigma_{k}\left(\lambda\left(D^{2} u\right)\right)=p(|x|) f_{2}(v), \\
\sigma_{l}\left(\lambda\left(D^{2} v\right)\right)=q(|x|) g_{1}(u) .
\end{array}\right.
$$

Related to $k$-Hessian equations, if $k=1$ the $k$-Hessian equations become the well-known Laplacian equations, and if $k=N$ the $k$-Hessian equations become the Monge-Ampère equations. Concerning Laplacian equations and Monge-Ampère equations, there are a great number of research papers, see for examples $[1,6,7,22]$ and the references therein. Here we specially mention Keller [15], Osserman [21], and Lair and Wood [17] for Laplacian equations and Cheng and Yau [2] and Laser and McKenna [19] for Monge-Ampère equations. Similar situations occur for coupling $k$-Hessian system (1.3), although in this case there are not so many research papers. Here we only mention Lair and Wood [18] and Cîrstea and Rădulescu [3] for coupling Laplacian systems and Wang and An [24] and Zhang and Qi [26] for coupling Monge-Ampère systems.

For general $k$-Hessian equation (1.2), when $p \equiv 1$ and $f(u)=u^{\gamma k}, \gamma>1$, Jin, Li, and Xu [13] showed the nonexistence of entire $k$-convex positive solutions. When $p \equiv 1$, Ji and Bao [11] gave necessary and sufficient conditions on the existence of entire positive $k$ convex radial solutions. If we generalize $p(|x|) f(u)$ to $f(x, u)$, de Oliveira, do Ó, and Ubilla obtained the existence of $k$-convex radial solutions in the case of supercritical nonlinearity by means of variational techniques (see [5] and the references therein for research in this direction). For general $k$-Hessian equation (1.2) and coupling $k$-Hessian system (1.3), Zhang and Zhou [27] obtained several results on the existence of entire positive $k$-convex radial solutions. We refer to the papers of Feng and Zhang [8] and Gao, He, and Ran [9] and the references therein for research on coupling $k$-Hessian system (1.3).

It is obvious that the $k$-Hessian type equation

$$
\sigma_{k}\left(\lambda\left(D^{2} u+\mu|\nabla u| I\right)\right)=p(|x|) f(u)
$$

is a generalization of $k$-Hessian equation (1.2), but it is a special case of the following fully nonlinear Hessian equation:

$$
F\left(\lambda\left(D^{2} u+A(x, u, \nabla u)\right)\right)=f(x, u, \nabla u)
$$

See Guan and Jiao [10] and Jiang and Trudinger [12] and the references therein for research on fully nonlinear Hessian equation (1.4). Here we also want to mention the work of Dai [4] for similar study.

Inspired by the works above, and as we know that now there are no papers on the problem of $k$-Hessian type system (1.1), we obtain the following results in this paper. 
Theorem 1.1 Under conditions $(H 1)$ and $(H 2)$, if $f_{1}(0) g_{2}(0) \neq 0$ and $f_{2}(0)+g_{1}(0) \neq 0$, then problem (1.1) admits an entire $k$-convex radial solution $(u, v) \in C^{2}\left(\overline{B_{1}(0)}\right) \times C^{2}\left(\overline{B_{1}(0)}\right)$.

Remark 1.1 In the case of $f_{1}(0) g_{2}(0)=0$, if $f_{1}(0)=g_{2}(0)=0$, then there is a trivial solution $(u, v)=(0,0)$ to problem $(1.1)$; if $f_{1}(0)=0$ or $g_{2}(0)=0$, then there is a semi-trivial solution $(u, v)=(0, v)$ or $(u, v)=(u, 0)$ to problem $(1.1)$; moreover, the semi-trivial solution may become trivial if $f_{1}(0)=0$ with $g_{1}(0)=0$ or $g_{2}(0)=0$ with $f_{2}(0)=0$.

In the case of $f_{2}(0)+g_{1}(0)=0$, there is a trivial solution $(u, v)=(0,0)$ to problem (1.1).

Theorem 1.2 Under conditions $(H 1)$ and (H3), problem (1.1) admits no entire $k$-convex radial solution $(u, v) \in C^{2}\left(\overline{B_{1}(0)}\right) \times C^{2}\left(\overline{B_{1}(0)}\right)$.

Remark 1.2 In this case, $f_{1}(0) f_{2}(0)=g_{1}(0) g_{2}(0)=0$, and there is a trivial solution $(u, v)=$ $(0,0)$ to problem $(1.1)$.

\section{Preliminaries}

In this section, we give some preliminary results which will be used to prove the main results in the next section.

Lemma 2.1 Assume $\varphi(r) \in C^{2}[0,1]$ with $\varphi^{\prime}(0)=0$. Then, for $u(x)=\varphi(r)$, there holds that $u \in C^{2}\left(\overline{B_{1}(0)}\right)$ and

$$
\lambda\left(D^{2} u+\eta|\nabla u| I\right)= \begin{cases}\left(\varphi^{\prime \prime}(r)+\eta \varphi^{\prime}(r),\left(\frac{1}{r}+\eta\right) \varphi^{\prime}(r), \ldots,\left(\frac{1}{r}+\eta\right) \varphi^{\prime}(r)\right), & r \in(0,1], \\ \left(\varphi^{\prime \prime}(0), \varphi^{\prime \prime}(0), \ldots, \varphi^{\prime \prime}(0)\right), & r=0,\end{cases}
$$

and further

$$
\begin{aligned}
\sigma_{k} & \left(\lambda\left(D^{2} u+\eta|\nabla u| I\right)\right) \\
& = \begin{cases}C_{N-1}^{k-1}\left(\varphi^{\prime \prime}(r)+\eta \varphi^{\prime}(r)\right)\left(\left(\frac{1}{r}+\eta\right) \varphi^{\prime}(r)\right)^{k-1}+C_{N-1}^{k}\left(\left(\frac{1}{r}+\eta\right) \varphi^{\prime}(r)\right)^{k}, & r \in(0,1], \\
C_{N}^{k}\left(\varphi^{\prime \prime}(0)\right)^{k}, & r=0,\end{cases}
\end{aligned}
$$

where $C_{N}^{k}=\frac{N !}{k !(N-k) !}$.

Proof It is immediate that, for $x \neq 0,1 \leq i, j \leq N$,

$$
\frac{\partial u(x)}{\partial x_{i}}=\left(\frac{\varphi^{\prime}(r)}{r}\right) x_{i}
$$

and

$$
\frac{\partial^{2} u(x)}{\partial x_{i} \partial x_{j}}=\left(\frac{\varphi^{\prime \prime}(r)}{r^{2}}\right) x_{i} x_{j}-\left(\frac{\varphi^{\prime}(r)}{r^{3}}\right) x_{i} x_{j}+\left(\frac{\varphi^{\prime}(r)}{r}\right) \delta_{i j} .
$$

Further if define

$$
\frac{\partial u(0)}{\partial x_{i}}=0, \quad \frac{\partial^{2} u(0)}{\partial x_{i} \partial x_{j}}=\varphi^{\prime \prime}(0) \delta_{i j}
$$

then $u \in C^{2}\left(\overline{B_{1}(0)}\right)$. 
Now it is easy to show the two equalities for $\lambda\left(D^{2} u+\eta|\nabla u| I\right)$ and $\sigma_{k}\left(\lambda\left(D^{2} u+\eta|\nabla u| I\right)\right)$

Lemma 2.2 Let $f \in C(-\infty, 0]$ be decreasing. Assume that $\varphi \in C^{0}[0,1] \cap C^{1}(0,1]$ is a solution of the Cauchy problem

$$
\left\{\begin{array}{l}
\varphi^{\prime}(r)=\left(\frac{k}{C_{N-1}^{k-1}} \mathrm{e}^{-\psi_{k, \eta}(r)} \int_{0}^{r} \mathrm{e}^{\psi_{k, \eta}(s)} \frac{s^{k-1} p(s)}{(1+\eta s)^{k-1}} f(\varphi(s)) d s\right)^{\frac{1}{k}}, \quad 0<r<1 \\
\varphi(1)=0
\end{array}\right.
$$

where

$$
\psi_{k, \eta}(r)=\frac{k}{C_{N-1}^{k-1}}\left(C_{N}^{k} \eta r+C_{N-1}^{k} \ln r\right)
$$

Then $\varphi \in C^{2}[0,1]$, and it satisfies the problem

$$
\left\{\begin{array}{l}
C_{N-1}^{k-1} \varphi^{\prime \prime}(r)\left(\varphi^{\prime}(r)\right)^{k-1} r+\left(C_{N}^{k} \eta r+C_{N-1}^{k}\right)\left(\varphi^{\prime}(r)\right)^{k}=\frac{r^{k} p(r)}{(1+\eta r)^{k-1}} f(\varphi(r)), \quad 0<r<1, \\
\varphi^{\prime}(0)=0 .
\end{array}\right.
$$

Furthermore, if $\varphi$ is nontrivial, i.e., $\varphi(r)<0$ for $0 \leq r<1$, then

$$
\lambda_{r}:=\left(\varphi^{\prime \prime}(r)+\eta \varphi^{\prime}(r),\left(\frac{1}{r}+\eta\right) \varphi^{\prime}(r), \ldots,\left(\frac{1}{r}+\eta\right) \varphi^{\prime}(r)\right) \in \Gamma_{k}
$$

for $0 \leq r<1$.

Proof It is easy to see that $\varphi(r) \in C^{2}[0,1]$.

From

$$
\varphi^{\prime}(r)=\left(\frac{k}{C_{N-1}^{k-1}} \mathrm{e}^{-\psi_{k, \eta}(r)} \int_{0}^{r} \mathrm{e}^{\psi_{k, \eta}(s)} \frac{s^{k-1} p(s)}{(1+\eta s)^{k-1}} f(\varphi(s)) d s\right)^{\frac{1}{k}}
$$

we have

$$
\left(\varphi^{\prime}(r)\right)^{k}=\frac{k}{C_{N-1}^{k-1}} \mathrm{e}^{-\psi_{k, \eta}(r)} \int_{0}^{r} \mathrm{e}^{\psi_{k, \eta}(s)} \frac{s^{k-1} p(s)}{(1+\eta s)^{k-1}} f(\varphi(s)) d s,
$$

and further differentiating with respect to $r$ we have

$$
C_{N-1}^{k-1} \varphi^{\prime \prime}(r)\left(\varphi^{\prime}(r)\right)^{k-1} r+\left(C_{N}^{k} \eta r+C_{N-1}^{k}\right)\left(\varphi^{\prime}(r)\right)^{k}=\frac{r^{k} p(r)}{(1+\eta r)^{k-1}} f(\varphi(r))
$$

If $\varphi$ is nontrivial, it is easy to see that $\varphi$ is increasing, so for $0 \leq r<1$ we conclude $\varphi(r)<$ $\varphi(1)=0, f(\varphi(r))>f(\varphi(1)) \geq 0$ and further

$$
\sigma_{k}\left(\lambda_{r}\right)=f(\varphi(r))>0 \quad \text { for } 0 \leq r<1
$$

By the properties of $k$ th elementary symmetric functions (see for example [20]), we know $\sigma_{j}\left(\lambda_{r}\right)>0$ for $1 \leq j<k$ and $0 \leq r<1$. Therefore we conclude the lemma. 


\section{Proofs of the main results}

In this section, we prove the main results in this paper, i.e., the existence and nonexistence of entire $k$-convex radial solutions for problem (1.1).

Proof of Theorem 1.1 From the system

$$
\left\{\begin{array}{l}
C_{N-1}^{k-1} u^{\prime \prime}(r)\left(u^{\prime}(r)\right)^{k-1} r+\left(C_{N}^{k} \mu r+C_{N-1}^{k}\right)\left(u^{\prime}(r)\right)^{k}=\frac{r^{k} p(r)}{(1+\mu r)^{k-1}} f_{1}(u(r)) f_{2}(v(r)), \\
C_{N-1}^{l-1} v^{\prime \prime}(r)\left(v^{\prime}(r)\right)^{l-1} r+\left(C_{N}^{l} \nu r+C_{N-1}^{l}\right)\left(v^{\prime}(r)\right)^{l}=\frac{r^{l} q(r)}{(1+\nu r)^{l-1}} g_{1}(u(r)) g_{2}(v(r)),
\end{array}\right.
$$

we get

$$
\left\{\begin{array}{l}
u^{\prime}(r)=\left(\frac{k}{C_{N-1}^{k-1}} \mathrm{e}^{-\psi_{k, \mu}(r)} \int_{0}^{r} \mathrm{e}^{\psi_{k, \mu}(s)} \frac{s^{k-1} p(s)}{(1+\mu s)^{k-1}} f_{1}(u(s)) f_{2}(v(s)) d s\right)^{\frac{1}{k}}, \\
v^{\prime}(r)=\left(\frac{l}{C_{N-1}^{l-1}} \mathrm{e}^{-\psi_{l, v}(r)} \int_{0}^{r} \mathrm{e}^{\psi_{l, v}(s)} \frac{s^{l-1} q(s)}{(1+\nu s)^{l-1}} g_{1}(u(s)) g_{2}(v(s)) d s\right)^{\frac{1}{k}},
\end{array}\right.
$$

furthermore we have

$$
\left\{\begin{array}{l}
u(r)=\int_{1}^{r}\left(\frac{k}{C_{N-1}^{k-1}} \mathrm{e}^{-\psi_{k, \mu}(t)} \int_{0}^{t} \mathrm{e}^{\psi_{k, \mu}(s)} \frac{s^{k-1} p(s)}{(1+\mu s)^{k-1}} f_{1}(u(s)) f_{2}(v(s)) d s\right)^{\frac{1}{k}} d t, \\
v(r)=\int_{1}^{r}\left(\frac{l}{C_{N-1}^{l-1}} \mathrm{e}^{-\psi_{l, v}(t)} \int_{0}^{t} \mathrm{e}^{\psi_{l, v}(s)} \frac{s^{l-1} q(s)}{(1+\nu s)^{l-1}} g_{1}(u(s)) g_{2}(v(s)) d s\right)^{\frac{1}{k}} d t .
\end{array}\right.
$$

Define

$$
\mathcal{L}(u, v)(r)=\left(\begin{array}{c}
\int_{1}^{r}\left(\frac{k}{C_{N-1}^{k-1}} \mathrm{e}^{-\psi_{k, \mu}(t)} \int_{0}^{t} \mathrm{e}^{\psi_{k, \mu}(s)} \frac{s^{k-1} p(s)}{(1+\mu s)^{k-1}} f_{1}(u(s)) f_{2}(v(s)) d s\right)^{\frac{1}{k}} d t \\
\int_{1}^{r}\left(\frac{l}{C_{N-1}^{l-1}} \mathrm{e}^{-\psi_{l, \nu}(t)} \int_{0}^{t} \mathrm{e}^{\psi_{l, \nu}(s)} \frac{s^{l-1} q(s)}{(1+\nu s)^{l-1}} g_{1}(u(s)) g_{2}(v(s)) d s\right)^{\frac{1}{k}} d t
\end{array}\right)^{T}
$$

then we need only to find a fixed point of $\mathcal{L}$. Here we use the monotone iterative method to find such a fixed point.

It is easy to show that $\mathcal{L}$ is a mapping from $C^{2}[0,1] \times C^{2}[0,1]$ to $C^{2}[0,1] \times C^{2}[0,1]$, and it is continuous on $C[0,1] \times C[0,1]$.

Let $\left\{u_{n}\right\}$ and $\left\{v_{n}\right\}$ be the sequence of continuous functions defined by

$$
\left\{\begin{array}{l}
u_{0}(r)=0, \\
v_{0}(r)=0, \\
u_{n}(r)=\int_{1}^{r}\left(\frac{k}{C_{N-1}^{k-1}} \mathrm{e}^{-\psi_{k, \mu}(t)} \int_{0}^{t} \mathrm{e}^{\psi_{k, \mu}(s)} \frac{s^{k-1} p(s)}{(1+\mu s)^{k-1}} f_{1}\left(u_{n-1}(s)\right) f_{2}\left(v_{n-1}(s)\right) d s\right)^{\frac{1}{k}} d t, \\
v_{n}(r)=\int_{1}^{r}\left(\frac{l}{C_{N-1}^{l-1}} \mathrm{e}^{-\psi_{l, \nu}(t)} \int_{0}^{t} \mathrm{e}^{\psi_{l, \nu}(s)} \frac{s^{l-1} q(s)}{(1+\nu s)^{l-1}} g_{1}\left(u_{n-1}(s)\right) g_{2}\left(v_{n-1}(s)\right) d s\right)^{\frac{1}{k}} d t .
\end{array}\right.
$$

It is easy to see that $u_{n}$ and $v_{n}$ are decreasing on $[0,1]$ for $n>1$ and by induction $\left\{u_{n}\right\}$ and $\left\{v_{n}\right\}$ are decreasing as well, i.e., $u_{n+1}(r)<u_{n}(r)$ and $v_{n+1}(r)<v_{n}(r)$ for $0 \leq r<1$ and $n \geq 1$.

By condition (H1), for each $0<r<1$ and $n>1$,

$$
\begin{aligned}
0 & <u_{n}^{\prime}(r) \\
& =\left(\frac{k}{C_{N-1}^{k-1}} \mathrm{e}^{-\psi_{k, \mu}(r)} \int_{0}^{r} \mathrm{e}^{\psi_{k, \mu}(s)} \frac{s^{k-1} p(s)}{(1+\mu s)^{k-1}} f_{1}\left(u_{n-1}(s)\right) f_{2}\left(v_{n-1}(s)\right) d s\right)^{\frac{1}{k}}
\end{aligned}
$$




$$
\begin{aligned}
& \leq C(N, k, p)\left(f_{1}\left(u_{n}(r)\right) f_{2}\left(v_{n}(r)\right)\right)^{\frac{1}{k}} \\
& \leq C(N, k, p)\left(f_{1}\left(u_{n}(r)+v_{n}(r)\right) f_{2}\left(u_{n}(r)+v_{n}(r)\right) d s\right)^{\frac{1}{k}},
\end{aligned}
$$

where $C(N, k, p)$ is a constant dependent on $N, k$, and $p$.

Similarly,

$$
0<v_{n}^{\prime}(r) \leq C(N, l, q)\left(g_{1}\left(u_{n}(r)+v_{n}(r)\right) g_{2}\left(u_{n}(r)+v_{n}(r)\right)\right)^{\frac{1}{l}}
$$

and further

$$
\begin{aligned}
0< & \left(u_{n}(r)+v_{n}(r)\right)^{\prime} \\
\leq & C(N, k, l, p, q)\left(\left(f_{1}\left(u_{n}(r)+v_{n}(r)\right) f_{2}\left(u_{n}(r)+v_{n}(r)\right)\right)^{\frac{1}{k}}\right. \\
& \left.+\left(g_{1}\left(u_{n}(r)+v_{n}(r)\right) g_{2}\left(u_{n}(r)+v_{n}(r)\right)\right)^{\frac{1}{l}}\right),
\end{aligned}
$$

i.e.,

$$
\begin{aligned}
0 & <\frac{\left(u_{n}(r)+v_{n}(r)\right)^{\prime}}{\left(f_{1}\left(u_{n}(r)+v_{n}(r)\right) f_{2}\left(u_{n}(r)+v_{n}(r)\right)\right)^{\frac{1}{k}}+\left(g_{1}\left(u_{n}(r)+v_{n}(r)\right) g_{2}\left(u_{n}(r)+v_{n}(r)\right)\right)^{\frac{1}{l}}} \\
& \leq C(N, k, l, p, q),
\end{aligned}
$$

where $C(N, l, q)$ and $C(N, k, l, p, q)$ are constants dependent on $N, l, q$ and $N, k, l, p, q$, respectively.

Integrating from 1 to $r$, we have

$$
\int_{0}^{u_{n}(r)+v_{n}(r)} \frac{d \tau}{\left(f_{1}(\tau) f_{2}(\tau)\right)^{\frac{1}{k}}+\left(g_{1}(\tau) g_{2}(\tau)\right)^{\frac{1}{l}}} \geq-C(N, k, l, p, q) .
$$

By condition (H2), denote

$$
F(w)=\int_{0}^{w} \frac{d \tau}{\left(f_{1}(\tau) f_{2}(\tau)\right)^{\frac{1}{k}}+\left(g_{1}(\tau) g_{2}(\tau)\right)^{\frac{1}{l}}},
$$

then $F$ is continuous and increasing on $(-\infty, 0]$, and it has an inverse function $F^{-1}$. From (3.2), we have

$$
\left.F^{-1}(-C(N, k, l, p, q)) \leq u_{n}(r)+v_{(} r\right) \leq 0
$$

for $0 \leq r \leq 1$ and $n \geq 1$.

By condition (H1) and (3.1), we have for $n \geq 1$

$$
\begin{aligned}
0 & <\left(u_{n}(r)+v_{n}(r)\right)^{\prime} \\
& \leq C(N, k, l, p, q)\left(\max _{F^{-1}(-C(N, k, l, p, q)) \leq w \leq 0}\left(\left(f_{1}(w) f_{2}(w)\right)^{\frac{1}{k}}+\left(g_{1}(w) g_{2}(w)\right)^{\frac{1}{T}}\right)\right) \\
& =C\left(N, k, l, p, q, f_{1}, f_{2}, g_{1}, g_{2}\right),
\end{aligned}
$$


where $C\left(N, k, l, p, q, f_{1}, f_{2}, g_{1}, g_{2}\right)$ is a constant dependent on $N, k, l, p, q, f_{1}, f_{2}, g_{1}$, and $g_{2}$. So $\left\{u_{n}\right\}$ and $\left\{v_{n}\right\}$ are bounded in $C^{1}[0,1]$ and by Arzela-Ascoli theorem $\left\{u_{n}\right\}$ and $\left\{v_{n}\right\}$ have convergent subsequences (still denoted by $\left\{u_{n}\right\}$ and $\left\{v_{n}\right\}$ ) in $C[0,1]$. Denote

$$
\begin{aligned}
& u(r)=\lim _{n \rightarrow+\infty} u_{n}(r), \\
& v(r)=\lim _{n \rightarrow+\infty} v_{n}(r) .
\end{aligned}
$$

By the continuity of $\mathcal{L}$ on $C[0,1] \times C[0,1]$, from

$$
\left(u_{n}, v_{n}\right)=\mathcal{L}\left(u_{n-1}, v_{n-1}\right),
$$

we conclude that $(u, v)$ is a fixed point of $\mathcal{L}$ after letting $n \rightarrow+\infty$.

Proof of Theorem 1.2 We prove by contradiction. Suppose that $(u, v)$ is a $k$-convex radial solution to problem (1.1). Then $u$ and $v$ are decreasing on [0,1]. For $0<r<1$, by Lemma 2.2 we can get

$$
\begin{aligned}
& 0<u^{\prime}(r) \leq C(N, k, p)\left(f_{1}(u(r)+v(r)) g_{2}(u(r)+v(r))\right)^{\frac{1}{k}}, \\
& 0<v^{\prime}(r) \leq C(N, l, q)\left(g_{1}(u(r)+v(r)) g_{2}(u(r)+v(r))\right)^{\frac{1}{T}} .
\end{aligned}
$$

So

$$
\begin{aligned}
0 & <\frac{(u(r)+v(r))^{\prime}}{\left(f_{1}(u(r)+v(r)) f_{2}(u(r)+v(r))\right)^{\frac{1}{k}}+\left(g_{1}(u(r)+v(r)) g_{2}(u(r)+v(r))\right)^{\frac{1}{l}}} \\
& \leq C(N, k, l, p, q) .
\end{aligned}
$$

Integrating for 0 to 1 , we have

$$
0<\int_{u(0)+v(0)}^{0} \frac{d \tau}{\left(f_{1}(\tau) f_{2}(\tau)\right)^{\frac{1}{k}}+\left(g_{1}(\tau) g_{2}(\tau)\right)^{\frac{1}{l}}} \leq C(N, k, l, p, q)
$$

which contradicts condition (H3). Now we finish the proof.

At the end of this section, we give some examples for the sake of clearly understanding the results in this paper.

Assume that $\alpha, \beta, \alpha_{1}, \beta_{1}, \alpha_{2}$, and $\beta_{2}$ are positive.

Example 3.1 If $\alpha_{1}+\beta_{1} \leq k$ and $\alpha_{2}+\beta_{2} \leq l$, then the following problem admits an entire $k$-convex radial solution $(u, v) \in C^{2}\left(\overline{B_{1}(0)}\right) \times C^{2}\left(\overline{B_{1}(0)}\right)$ :

$$
\begin{cases}\sigma_{k}\left(\lambda\left(D^{2} u+\mu|\nabla u| I\right)\right)=(1+|x|)^{\alpha}(1+|u|)^{\alpha_{1}}|v|^{\beta_{1}}, & x \in B_{1}(0), \\ \sigma_{l}\left(\lambda\left(D^{2} v+v|\nabla v| I\right)\right)=(1+|x|)^{\beta}(1+|u|)^{\alpha_{2}}(1+|v|)^{\beta_{2}}, & x \in B_{1}(0), \\ u=v=0, & x \in \partial B_{1}(0) .\end{cases}
$$


Example 3.2 If $\alpha_{1}+\beta_{1} \geq k$ and $\alpha_{2}+\beta_{2} \geq l$, then the following problem admits no entire $k$-convex radial solution $(u, v) \in C^{2}\left(\overline{B_{1}(0)}\right) \times C^{2}\left(\overline{B_{1}(0)}\right)$ :

$$
\begin{cases}\sigma_{k}\left(\lambda\left(D^{2} u+\mu|\nabla u| I\right)\right)=(1+|x|)^{\alpha}|u|^{\alpha_{1}}|v|^{\beta_{1}}, & x \in B_{1}(0), \\ \sigma_{l}\left(\lambda\left(D^{2} v+v|\nabla v| I\right)\right)=(1+|x|)^{\beta}|u|^{\alpha_{2}}|v|^{\beta_{2}}, & x \in B_{1}(0), \\ u=v=0, & x \in \partial B_{1}(0) .\end{cases}
$$

\section{Conclusion}

In this paper, by converting the existence of an entire solution to the existence of a fixed point of a continuous mapping, we establish the existence of entire $k$-convex radial solutions for a Hessian type system. Moreover the nonexistence of entire $k$-convex radial solutions is also obtained. In the process of obtaining the existence of entire $k$-convex radial solutions, we utilize the monotone iterative method. By different fixed point theorems (such as the ones in [14] and [25]) or different methods (such as degree theory in [16] and the regularization method in [23]), we may get different results on Hessian type systems. In our opinion, it is interesting to fulfil this kind of works in the future.

\section{Acknowledgements}

The author is grateful to the referees for their helpful remarks and suggestions.

\section{Funding}

I am supported by the National Natural Science Foundation of China (Grant No. U2031142).

Availability of data and materials

No data were used to support this study. It is not applicable for my paper.

\section{Competing interests}

The author declares that he has no competing interests.

\section{Authors' contributions}

The author contributed independently to the manuscript and read and approved the final manuscript.

\section{Publisher's Note}

Springer Nature remains neutral with regard to jurisdictional claims in published maps and institutional affiliations.

Received: 19 February 2021 Accepted: 22 September 2021 Published online: 18 October 2021

\section{References}

1. Cavalheiro, A.C.: Existence results for Navier problems with degenerated $(p, q)$-Laplacian and $(p, q)$-biharmonic operators. Results Nonlinear Anal. 1(2), 74-87 (2018)

2. Cheng, S.Y., Yau, S.T.: On the existence of a complete Kähler metric on noncompact complex manifolds and the regularity of Fefferman's equation. Commun. Pure Appl. Math. 33, 507-544 (1980)

3. Cîrstea, F.C., Rădulescu, V.: Entire solutions blowing up at infinity for semilinear elliptic systems. J. Math. Pures Appl. 81, 827-846 (2002)

4. Dai, L.M.: Existence and nonexistence of subsolutions for augmented Hessian equations. Discrete Contin. Dyn. Syst. 40(1), 579-596 (2020)

5. de Oliveira, J.F., do Ó, J.M., Ubilla, P.: Existence for a k-Hessian equation involving supercritical growth. J. Differ. Equ. 267, 1001-1024 (2019)

6. Enache, C., Porru, G.: A note on Monge-Ampere equation in $\mathbb{R}^{2}$. Results Math. 76(1), Article ID 29 (2021)

7. Feng, M.Q:: Convex solutions of Monge-Ampère equations and systems: existence, uniqueness and asymptotic behavior. Adv. Nonlinear Anal. 10(1), 371-399 (2021)

8. Feng, M.Q., Zhang, X.M.: A coupled system of k-Hessian equations. Math. Methods Appl. Sci. 44(9), 7377-7394 (2021)

9. Gao, C.H., He, X.Y., Ran, M.J.: On a power-type coupled system of k-Hessian equations. Quaest. Math. https://doi.org/10.2989/16073606.2020.1816586

10. Guan, B., Jiao, H.: Second order estimates for Hessian type fully nonlinear elliptic equations on Riemannian manifolds. Calc. Var. Partial Differ. Equ. 54, 2693-2712 (2015)

11. Ji, X., Bao, J.: Necessary and sufficient conditions on solvability for Hessian inequalities. Proc. Am. Math. Soc. 138, $175-188(2010)$

12. Jiang, F., Trudinger, N.S.: On the Dirichlet problem for general augmented Hessian equations. J. Differ. Equ. 269, 5204-5227 (2020) 
13. Jin, Q., Li, Y., Xu, H.: Nonexistence of positive solutions for some fully nonlinear elliptic equations. Methods Appl. Anal. $12,441-450(2005)$

14. Karapınar, E.: A fixed point theorem without a Picard operator. Results Nonlinear Anal. 4(3), 127-129 (2021)

15. Keller, J.B.: On solutions of $\Delta u=f(u)$. Commun. Pure Appl. Math. 10, 503-510 (1957)

16. Kim, I.S.: Semilinear problems involving nonlinear operators of monotone type. Results Nonlinear Anal. 2(1), 25-35 (2019)

17. Lair, A.V., Wood, A.W.: Large solutions of semilinear elliptic problems. Nonlinear Anal. 37, 805-812 (1999)

18. Lair, A.V., Wood, A.W.: Existence of entire large positive solutions of semilinear elliptic systems. J. Differ. Equ. 164, 380-394 (2000)

19. Lazer, A.C., McKenna, P.J.: On singular boundary value problems for the Monge-Ampère operator. J. Math. Anal. Appl. 197, 341-362 (1996)

20. Lieberman, G.: Second Order Parabolic Differential Equations. World Scientific, New Jersey (1996)

21. Osserman, R.: On the inequality $\Delta u \geq f(u)$. Pac. J. Math. 7, 1641-1647 (1957)

22. Ourraou, A.: Existence and uniqueness of solutions for Steklov problem with variable exponent. Adv. Theory Nonlinear Anal. Appl. 5(1), 158-166 (2021)

23. Phuong, N.D., Luc, N.H., Long, L.D.: Modified quasi boundary value method for inverse source problem of the bi-parabolic equation. Adv. Theory Nonlinear Anal. Appl. 4(3), 132-142 (2020)

24. Wang, F., An, Y.: Triple nontrivial radial convex solutions of systems of Monge-Ampère equations. Appl. Math. Lett. 25, 88-92 (2012)

25. Zhang, C., Chen, J.: Convergence analysis of variational inequality and fixed point problems for pseudo-contractive mapping with Lipschitz assumption. Results Nonlinear Anal. 2(3), 102-112 (2019)

26. Zhang, Z., Qi, Z.: On a power-type coupled system of Monge-Ampère equations. Topol. Methods Nonlinear Anal. 46, 717-729 (2015)

27. Zhang, Z., Zhou, S.: Existence of entire positive $k$-convex radial solutions to Hessian equations and systems with weights. Appl. Math. Lett. 50, 48-55 (2015)

\section{Submit your manuscript to a SpringerOpen ${ }^{\circ}$ journal and benefit from:}

- Convenient online submission

- Rigorous peer review

- Open access: articles freely available online

- High visibility within the field

- Retaining the copyright to your article

Submit your next manuscript at $\gg$ springeropen.com 